\title{
Factores asociados con la letalidad por neumonía en una unidad de atención del paciente geriátrico agudo: una cohorte retrospectiva
}

\author{
Diana C. Quintero-González1, José Mauricio Ocampo,3, Carlos A. Reyes-Ortiz \\ ${ }^{1}$ Departamento de Medicina interna, Universidad Libre, seccional Cali, Cali, Colombia \\ ${ }^{2}$ Universidad del Valle, Facultad de Salud, Departamento de Medicina Familiar, Cali, Colombia \\ ${ }^{3}$ Grupo Interinstitucional de Medicina Interna, Departamento de Medicina Interna, Facultad \\ Ciencias de la Salud, Universidad Libre, Cali, Colombia \\ ${ }^{4}$ Institute of Public Health, College of Pharmacy and Pharmaceutical Sciences, Florida A \& M \\ University, Tallahassee, FL, USA
}

Introducción. La neumonía es la principal causa de muerte por infección en el mundo y afecta principalmente a la población de adultos mayores.

Objetivo. Determinar los factores de riesgo asociados con la letalidad a 30 días en pacientes con neumonía en una unidad de atención del paciente geriátrico agudo. Materiales y métodos. Se hizo un estudio observacional y analítico de cohorte retrospectiva. Se incluyeron 114 pacientes de 60 años o más hospitalizados en una unidad de atención del paciente geriátrico agudo con diagnóstico de neumonía. El resultado primario fue la letalidad a 30 días. Se hicieron análisis de regresión log-binomial bivariado y multivariable para explorar la relación entre las variables independientes y el resultado primario.

Resultados. La letalidad a 30 días fue de $26,3 \%$ y la edad media de $84,45 \pm 7,37$ años. El $54,4 \%$ de los participantes era de sexo masculino. En el análisis multivariable se encontró que una edad de 90 años o más (riesgo relativo, $R R=1,62 ; I_{95 \%}: 1,05-2,68$; $\mathrm{p}=0,04)$, el compromiso multilobar ( $\left.\mathrm{RR}=1,92 ; \mathrm{IC}_{95 \%}: 1,12-3,32 ; \mathrm{p}=0,02\right)$, el nitrógeno ureico elevado (mediana $\geq 22,5 ; \mathrm{RR}=3,93 ; \mathrm{IC}_{95 \%}: 1,67-9,25 ; \mathrm{p}<0,01$ ), y un puntaje de cero en la escala de Lawton al ingreso $\left(R R=3,20 ; i C_{95 \%} 1,05-9,78 ; p=0,04\right)$ fueron factores predictores independientes de la letalidad a 30 días en adultos mayores con neumonía.

Recibido: 09/11/2019

Aceptado: $29 / 07 / 2020$

Publicado: 06/08/2020

Citación:

Quintero-González DC, Ocampo JM, Reyes-Ortiz CA. Factores asociados con la letalidad por neumonía en una unidad de atención del paciente geriátrico agudo: una cohorte retrospectiva. Biomédica. 2020;40(Supl.2):734-48.

https://doi.org/10.7705/biomedica.5244

\section{Correspondencia:}

Carlos A. Reyes-Ortiz, Institute of Public Health, College of Pharmacy and Pharmaceutical Sciences, Florida A \& M University; 1515 South MLK Blvd., Suite 209D, Tallahassee, FL 32307, USA Teléfono: (850) 5612882; fax: (850) 5998830 careyesortiz@ hotmail.com

Contribución de los autores:

José Mauricio Ocampo: adquisición de datos Todos los autores participaron en la concepción y diseño del estudio, el planteamiento y estructuración de la pregunta de investigación, el diseño y elaboración del análisis estadístico, la interpretación y descripción de resultados, la revisión sistemática de la literatura y la escritura del manuscrito.

\section{Financiación:}

Esta investigación no recibió ningún tipo de financiamiento por parte del sector público o privado.

Conflicto de intereses:

Los autores declaran no tener ningún conflicto de intereses.
Conclusión. En los pacientes adultos mayores hospitalizados por neumonía en una unidad de atención del paciente geriátrico agudo la edad muy avanzada, la presencia de compromiso multilobar, la dependencia en el estado funcional y los niveles de nitrógeno ureico elevados fueron los principales factores de riesgo de letalidad a corto plazo.

Palabras clave: neumonía; anciano; mortalidad; radiografía; nitrógeno de la urea sanguínea; evaluación de la discapacidad.

Factors associated with lethality from pneumonia in an acute care for the elderly unit: a retrospective cohort

Introduction: Pneumonia is the leading cause of death due to infection in the world, mainly affecting the older adult population.

Objective: To determine the risk factors associated with lethality at 30 days in patients with pneumonia in an acute care for the elderly unit.

Materials and methods: We conducted an observational, analytical retrospective cohort study including 114 patients aged 60 years and older hospitalized in an acute care geriatric unit with a diagnosis of pneumonia. The primary outcome was lethality at 30 days. Bivariate and multivariable log-binomial regression analyzes were performed to explore the relationship between independent variables and the primary outcome.

Results: The 30 -day lethality was $26.3 \%$ and the mean age was $84.45 \pm 7.37$ years; $54.4 \%$ of participants were men. In the multivariable analysis, age $\geq 90$ years (Relative Risk, $\mathrm{RR}=1.62,95 \% \mathrm{Cl}: 1.05-2.68, \mathrm{p}=0.04)$, multilobar commitment $(\mathrm{RR}=1.92,95 \% \mathrm{Cl}: 1.12-3.32$ $p=0.02$ ), elevated urea nitrogen ( $\geq 22.5$ the median; $R R=3.93,95 \% \mathrm{Cl}: 1.67-9.25, p<0.01$ ), and a score of zero in the Lawton index at admission ( $R R=3.20,95 \% \mathrm{Cl}: 1.05-9.78, p=0.04)$ were independent predictors of 30-day lethality from pneumonia.

Conclusion: In older adults hospitalized for pneumonia in an acute care geriatric unit, advanced age, the presence of multilobar commitment, dependency in functional status, and elevated ureic nitrogen levels were the main predictors of short-term lethality risk.

Keywords: Pneumonia; aged; mortality; radiography; blood urea nitrogen; disability evaluation. 
La neumonía es un proceso inflamatorio del parénquima pulmonar de tipo infeccioso que puede ocurrir cuando la virulencia del agente patógeno sobrepasa las defensas del huésped $(1,2)$. Los adultos mayores son uno de los grupos etarios más susceptibles de desarrollar la neumonía (3). Según la Organización Panamericana de la Salud (OPS), adulto mayor se define como una persona de 60 años o más (4). La población de adultos mayores en los servicios de urgencias ha aumentado, lo cual se debe al incremento uniforme y acelerado de este grupo en todo el mundo. En Europa se espera que para el 2060 la población de 65 años o más aumente del 18 al 28 \% (5). Una situación similar se evidencia en Colombia, donde el porcentaje de mujeres y hombres adultos mayores aumentó de 7,3 y $6,7 \%$ en 1985 hasta 13,8 y $11,5 \%$ en el 2020, respectivamente (6).

A nivel mundial, la incidencia de neumonía adquirida en la comunidad tiene forma de $\mathrm{U}$, pues afecta principalmente a los menores de 5 años y a los mayores de 65 (3). En los países europeos, la incidencia de neumonía en la comunidad puede ser 10,8 veces mayor en las personas de 85 años o más comparada con personas de 50 a 64 años (7). En los Estados Unidos se estima que anualmente hay 1,3 millones de casos nuevos de neumonía (8); además, se ha demostrado que la tasa de incidencia de la neumonía (847 casos por cada 100.000 personas/año) es superior a la de las enfermedades no transmisibles frecuentes como el infarto de miocardio y la enfermedad cerebrovascular (9). En Colombia, un estudio del 2009 encontró que la tasa de incidencia de neumonía en pacientes ambulatorios y hospitalizados de 50 años o mayores fue de 336 y 327 casos por cada 100.000 personas/año, respectivamente (10). Además, la incidencia de neumonía en pacientes hospitalizados aumentó de 326 casos por cada 100.000 personas/año en el grupo de 65 a 74 años de edad a 4.636 casos en personas de 85 años o mayores (10).

Por otro lado, la neumonía se cataloga como la principal causa de muerte de origen infeccioso en el mundo (11). En los Estados Unidos se ha reportado que la letalidad en pacientes hospitalizados es dos veces mayor en aquellos de 75 años o mayores que en los de 65 a 74 años (10,6 \% Vs. 4,9\%) (12). Un estudio observacional realizado en seis países latinoamericanos encontró que la letalidad media en pacientes de 50 años o mayores hospitalizados por neumonía fue de $17,7 \%$, en tanto que en Colombia fue del $11 \%$ en el 2009 (10). Además, en el país, la letalidad fue mayor en personas de 65 a 74 años y en las de 85 años o más (9,5\% y 13,6\%, respectivamente) (10). Asimismo, según las estadísticas del Departamento Administrativo Nacional de Estadística (DANE), las infecciones respiratorias agudas constituyeron el $4,23 \%$ de las causas de muerte entre el 2016 y el 2017, ocupando el quinto lugar (13). Además, se ha estimado que la letalidad hospitalaria de los adultos mayores en general puede llegar a ser del $19 \%$ (14).

Las unidades de atención del paciente geriátrico agudo se han diseñado para prevenir la declinación funcional y las complicaciones por yatrogenia en el adulto mayor por medio de un modelo de atención integral (15). Aunque su efectividad ha sido demostrada por la disminución de las caídas, el delirio, la declinación funcional y la duración de la hospitalización, no se ha descrito si su impacto en la tasa de letalidad es similar (16).

De hecho, la neumonía se ha descrito como la primera causa de muerte en estas unidades, con tasas elevadas que oscilan entre el 37,5 y el $40 \%$ (17). A pesar de las opciones de tratamiento, la vacunación y la disponibilidad de pruebas diagnósticas, la letalidad por neumonía en los adultos mayores 
sigue siendo alta. En los últimos 10 años los factores que se han asociado con la letalidad por esta condición son la edad, un índice de comorbilidad de Charlson elevado, comorbilidades como la enfermedad pulmonar obstructiva crónica (EPOC), la enfermedad cerebrovascular, la fractura de cadera, las neoplasias sólidas, la malnutrición, el estado mental alterado, la hipotensión, el grado de dependencia, el compromiso bilateral y el derrame pleural (18-24).

En Colombia, son pocos los estudios que han investigado los factores asociados con la letalidad en la población de adultos mayores. El presente estudio pretende contribuir a cerrar esta brecha en el conocimiento, determinando los factores asociados con la letalidad a 30 días en pacientes con neumonía en una unidad de atención del paciente geriátrico agudo.

\section{Materiales y métodos}

\section{Diseño del estudio y recolección de la información}

Se hizo un estudio observacional de cohorte retrospectiva de 114 pacientes con edad igual o mayor a 60 años y diagnóstico de neumonía hospitalizados en una unidad de atención del paciente geriátrico agudo entre marzo de 2011 y agosto de 2015.

Para ingresar a la unidad, los pacientes debían tener una edad igual o mayor a 60 años y cumplir con uno o más de los siguientes criterios: múltiples comorbilidades (dos o más), condición funcional previa de dependencia grave o total, hospitalizaciones frecuentes (dos o más al mes), demencia previa o delirio al ingreso, presencia de dos o más síndromes geriátricos (por ejemplo, fragilidad, úlceras por presión o caídas), índice de masa corporal menor de $20 \mathrm{~kg} / \mathrm{m}^{2}$, red de apoyo social insuficiente, proceder de instituciones geriátricas, o tener más de 80 años con enfermedad aguda.

Para el diagnóstico de neumonía se consideraron los criterios publicados en la guía colombiana de 2013. Los pacientes debían cumplir con, al menos, dos de los siguientes signos y síntomas: temperatura mayor o igual a 38,3 $\mathrm{C}$, tos productiva, dolor torácico, disnea o crepitación en la auscultación, además de una nueva opacidad en la radiografía de tórax (1). Se excluyeron los pacientes con antecedente de infección por el virus de inmunodeficiencia humana $(\mathrm{VIH})$, tuberculosis activa y aquellos con variables clínicas no incluidas en la historia clínica. Los datos analizados ya estaban recolectados y se seleccionó esta cohorte de forma retrospectiva.

\section{Variables de estudio}

Resultado. Consistió en la letalidad a 30 días posterior al diagnóstico de neumonía en pacientes hospitalizados en una unidad de atención del paciente geriátrico agudo. La información de letalidad se recolectó de las historias clínicas y fue corroborada con las estadísticas vitales suministradas por la Secretaría de Salud Departamental del Valle del Cauca mediante los certificados de defunción hasta septiembre del 2015. Los códigos CIE10 descritos como causa directa de muerte fueron: A419, septicemia no especificada; J159, neumonía bacteriana no especificada; J960, insuficiencia respiratoria aguda, y J969, insuficiencia respiratoria no especificada.

Variables independientes. Las variables sociodemográficas incluían la edad, el sexo y la escala de valoración sociofamiliar de Gijón (menor a 8 puntos: sin riesgo social). Las variables clínicas fueron la hospitalización en días; el índice de comorbilidad de Charlson (sin comorbilidad: 1 punto, 
comorbilidad baja: 2 puntos, comorbilidad alta: 3 o más puntos) (25); el número de medicamentos al ingreso, y la presencia de las siguientes comorbilidades: hipertensión, enfermedad cerebrovascular, diabetes mellitus, dislipidemia, EPOC, enfermedad renal crónica, fibrilación auricular, demencia, hipotiroidismo, trastorno de la deglución, insuficiencia cardiaca, epilepsia, cáncer y tabaquismo.

En cuanto al examen físico, se incluyó la frecuencia cardiaca (latidos por minuto), la frecuencia respiratoria (respiraciones por minuto), la hipotensión sistólica definida como una tensión arterial sistólica menor o igual a $90 \mathrm{~mm}$ $\mathrm{Hg}$, y la hipoxemia definida como oximetría menor o igual a $90 \%$ o presión arterial de oxígeno menor o igual a $60 \mathrm{~mm} \mathrm{Hg}$ en gases arteriales.

En cuanto a las variables de laboratorio y de imagenología, se incluyeron la presencia de derrame pleural o compromiso multilobar (dos o más lóbulos pulmonares) en la radiografía de tórax, leucocitos (células $/ \mathrm{mm} 3$ ), hemoglobina $(\mathrm{g} / \mathrm{dl})$, albúmina $(\mathrm{mg} / \mathrm{dl})$, creatinina $(\mathrm{mg} / \mathrm{dl})$, nitrógeno ureico $(\mathrm{mg} / \mathrm{dl})$ empleado como variable numérica pero también categorizado como BUN (blood urea nitrigen) elevado de acuerdo a la mediana $(\geq 22,5 \mathrm{Vs}$. $<22,5)$ en la construcción de riesgo relativo, sodio $(\mathrm{mg} / \mathrm{dl})$, y proteína $\mathrm{C}$ reactiva (PCR; $\mathrm{mg} / \mathrm{dl})$.

El resultado de las variables de laboratorio correspondió a su primera medición en el momento del ingreso del paciente. Además, se calculó la escala CURB-65, la cual describe las siguientes variables: confusión, nitrógeno ureico mayor o igual a $20 \mathrm{mg} / \mathrm{dl}$, frecuencia respiratoria mayor o igual a 30 respiraciones por minuto, tensión arterial sistólica menor o igual a $90 \mathrm{~mm} \mathrm{Hg} \mathrm{y}$ edad mayor o igual a 65 años (26); cada variable suma un punto en la escala, o sea que a mayor número de puntos mayor la gravedad del cuadro. Con base en estos parámetros los pacientes se clasificaron en tres grupos de riesgo: grupo 1 (0 a 1 punto), grupo 2 ( 2 puntos) y grupo 3 (3 o más puntos).

Para la evaluación de la capacidad funcional en las actividades básicas de la vida diaria se tomó el índice de Barthel al ingreso (27), considerando a un paciente como dependiente si obtenía un puntaje menor o igual a 60 puntos. También se incluyó la evaluación de las actividades instrumentales de la vida diaria mediante la escala de Lawton y Brody en el momento de ingreso (28), categorizando a los pacientes como totalmente dependientes (0 puntos) o no (1 a 8 puntos). Por último, se documentó la presencia de delirio y deterioro cognitivo a través de la escala del Confusion Assessment Method (CAM) (29) y la escala Mini-Mental, respectivamente (30), así como el esquema antibiótico y el número de días de tratamiento.

\section{Análisis estadístico}

Se empleó estadística descriptiva para resumir las características de la población. Las variables cuantitativas se expresaron en medidas de tendencia central y dispersión. Para evaluar la distribución de los datos se usó la prueba de Kolmogórov-Smirnov y se asumió como significativos los valores de $p<0,05$. Las variables cualitativas se resumieron como proporciones y se presentaron en tablas de frecuencia. La significación estadística de las asociaciones en las variables numéricas se determinó con la prueba $U$ de Mann Whitney, y la de las variables dicotómicas con ji al cuadrado o la prueba de Fisher según correspondiera; para rechazar las hipótesis nulas se asumieron como significativos valores de $p<0,05$. También se hizo un análisis de sensibilidad para evaluar si los excluidos eran significativamente diferentes de los participantes. 
Para explorar la presencia de asociaciones entre la variable de resultado (letalidad a 30 días) y las variables de exposición, se hicieron los análisis de regresión log binomial bivariado y multivariable. Para ello se usó el Genmod Procedure en SAS ${ }^{\mathrm{TM}}$, el cual utiliza la distribución de Poisson para estimar los riesgos relativos (RR) con intervalos de confianza (IC) del $95 \%$.

Para ajustar los posibles factores predictores se utilizó un modelo de regresión multivariable. Para la selección de dicho modelo se usó el criterio modificado de Greenland (31). Inicialmente, se seleccionaron aquellas variables con $p<0,20$ asociados tanto con la variable dependiente (letalidad) como con la principal variable independiente (elegimos el BUN, ya que en otro estudio la función renal baja se encontró asociada con una mayor mortalidad) (32). Las que produjeron una variación significativa igual o mayor del $10 \%$ en la asociación entre la variable dependiente y la principal, se mantuvieron en el modelo multivariable. Para el diagnóstico de este modelo se usaron parámetros tales como la convergencia, la multicolinealidad y la sobredispersión. La bondad del ajuste se evaluó con la prueba de la desviación, la cual mostró muy buen ajuste (grados de libertad=109; valor=54,66; $p=0,99$ ).

Además, se calculó el riesgo relativo de letalidad como la razón de riesgo en los expuestos $(a /[a+b]$ son los pacientes que tienen uno o más factores de riesgo: edad $\geq 90$ años, hipoxemia, dependencia total en la escala de Lawton, BUN alto y compromiso multilobar) dividido por el riesgo en los no expuestos (c/[C+d] pacientes sin algún factor de riesgo) y se calcularon los $I_{95 \%}$. Los análisis estadísticos se realizaron en los programas Stata ${ }^{\mathrm{TM}}$ (Stata Corp, 2011, Stata 12 Base Reference Manual, College Station, TX, USA) y SASTM, versión 9.4 para Windows (SAS Institute, Inc., Cary, NC).

\section{Consideraciones éticas}

El protocolo del estudio primario, del cual se recolectó la información para este trabajo de investigación, contó con el consentimiento informado y las precauciones necesarias para garantizar la privacidad de los pacientes (la protección de los datos anonimizados y el manejo dado por el equipo investigador). El estudio fue aprobado por el Comité de Ética y Científico de la Universidad Libre y de la Clínica Universitaria Rafael Uribe Uribe.

\section{Resultados}

De 232 pacientes con diagnóstico principal o secundario de neumonía, 28 fueron excluidos por no tener información disponible sobre mortalidad y en 90 , porque la mayoría de las variables clínicas relacionadas con el desenlace no pudieron ser verificadas en la historia clínica (figura 1). Comparados con los pacientes que se incluyeron en el estudio $(n=114)$, los pacientes excluidos $(n=118)$ tenían más dependencia en cuanto a su estado funcional (con un puntaje en la escala de Lawton significativamente más bajo: $p=0,03$, y en el índice de Barthel: $p<0,01$ ), pero no tenían diferencias significativas de edad, sexo, comorbilidades, estado mental (MMSE) ni presencia de delirio. El resultado primario, es decir, la letalidad en los 30 días posteriores al diagnóstico, se presentó en 30 pacientes. La media de tiempo entre el diagnóstico y el desenlace fatal fue de $16 \pm 7,8$ días.

Las características sociodemográficas, funcionales, clínicas y de los exámenes de laboratorio se resumen en los cuadros 1 y 2 . Se encontró una media de edad superior en los pacientes con el resultado primario (87,2 años 
Vs. 83,5 años). No hubo diferencias significativas en la relación hombremujer, el estado sociofamiliar, la duración de la hospitalización en días, el índice de comorbilidad de Charlson y el número de medicamentos, entre otras descritas en el cuadro 1. La evaluación de la capacidad funcional en el momento de ingreso demostró que los pacientes del grupo donde hubo muertes tenían una mayor proporción de dependencia total según el índice de Barthel $(93,3 \%$ Vs. $63,1 \% ; p<0,01)$. Este hallazgo fue similar en la escala de Lawton y Brody, en el que el $90,0 \%$ del grupo que registró muertes tenía dependencia total (puntaje de cero) comparado con el 56,0 \% en el grupo de quienes no la tenían $(p<0,01)$. El porcentaje de delirio evaluado con la escala del Confusion Assessment Method fue de 46,7 \% Vs. 36,9\% $(p=0,35)$.

En el examen físico, la hipoxemia se presentó en el 73,3\% de los pacientes del grupo donde hubo muertes $(p=0,001)$. Según los resultados de laboratorio, la mediana y el rango intercuartílico $(\mathrm{RIC})$ de nitrógeno ureico fue de $34 \mathrm{mg} / \mathrm{dl}$ en el grupo en el que se registraron muertes a los 30 días (RIC: 25 - $54 \mathrm{mg} / \mathrm{dl})$ comparado con $20 \mathrm{mg} / \mathrm{dl}$ (RIC: 15 - 26,5mg/dl) $(\mathrm{p}<0,01)$ en el otro grupo; asimismo, el 83,3\% del grupo en el que hubo muertes tenían un BUN $\geq 22,5 \mathrm{mg} / \mathrm{dl}$ comparado con el $38,1 \%$ de los sobrevivientes $(p<0,01)$. No hubo diferencias en el número de leucocitos al ingreso o en los reactantes de fase aguda como la proteína $\mathrm{C}$ reactiva. El compromiso multilobar en la radiografía de tórax fue mayor en el grupo en el que hubo muertes $(13,3 \% V s .2,4 \%, p=0,04)$. La evaluación de la gravedad según la escala CURB65 demostró un porcentaje más alto de pacientes del grupo 3 en el grupo en el que hubo muertes (66,3\% Vs. $29,8 \%$; $p<0,01)$, lo que indica una mayor gravedad del cuadro. Los antibióticos más utilizados fueron los inhibidores de betalactamasas (ampicilina más sulbactam y piperacilina más tazobactam): 76,7 \% en el grupo en el que hubo muertes $V s .73,8 \%$ en los que sobrevivieron los primeros 30 días. No hubo diferencias significativas en el número de días de tratamiento $(p=0,7)$.

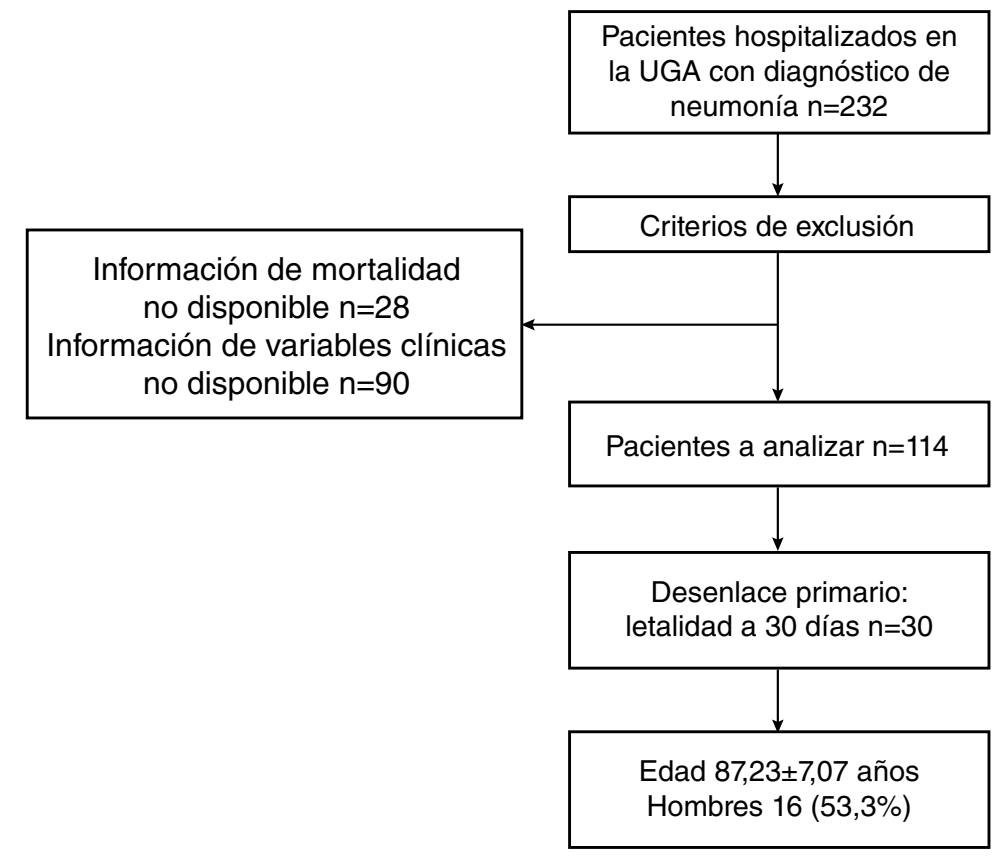

Figura 1. Pacientes de la unidad de atencion del paciente geriatrico agudo con diagnóstico de neumonía entre el 2011 y el 2015 
Cuadro 1. Características sociodemográficas y clínicas de la población de estudio

\begin{tabular}{|c|c|c|c|c|c|c|c|}
\hline \multirow[b]{2}{*}{ Características } & \multirow{2}{*}{\multicolumn{2}{|c|}{$\begin{array}{c}\text { General } \\
\begin{array}{c}n=114 \\
n(\%)\end{array}\end{array}$}} & \multicolumn{4}{|c|}{ Letalidad a 30 días } & \multirow[b]{2}{*}{$\mathbf{p}$} \\
\hline & & & \multicolumn{2}{|c|}{$\begin{array}{c}\text { Sí } \\
(n=30) \\
n(\%) \\
\end{array}$} & \multicolumn{2}{|r|}{$\begin{array}{c}\text { No } \\
(n=84) \\
n(\%)\end{array}$} & \\
\hline$\overline{\text { Edad }^{*}}$ & 84,4 & $45(7,37)$ & 87,2 & $23(7,07)$ & 83,4 & $5(7,25)$ & 0,02 \\
\hline Edad $\geq 90$ & 28 & $(24,6)$ & 12 & $(40,0)$ & 16 & $(19,0)$ & 0,02 \\
\hline \multicolumn{8}{|l|}{ Sexo } \\
\hline Masculino & 62 & $(54,39)$ & 16 & $(53,33)$ & 46 & $(54,76)$ & 0,89 \\
\hline Femenino & 52 & $(45,61)$ & 14 & $(46,67)$ & 38 & $(45,24)$ & \\
\hline \multicolumn{8}{|l|}{ Escala de Gijón } \\
\hline Sin riesgo social ${ }^{\triangleright}$ & 98 & $(85,96)$ & 28 & $(93,33)$ & 70 & $(83,33)$ & \\
\hline Con riesgo social & 16 & $(14,04)$ & 2 & $(6,67)$ & 14 & $(16,67)$ & $0,23 \mathrm{t}$ \\
\hline Hospitalización en días ${ }^{\star \star}$ & 12 & $(9-16)$ & 12,5 & $5(10-17)$ & 12 & $(9-15)$ & 0,37 \\
\hline Índice de Charlson** & 3 & $(2-4)$ & 3 & $(2-5)$ & 3 & $(2-4)$ & 0,48 \\
\hline Número de medicamentos al ingreso** & 5 & $(3-8)$ & 5 & $(3-8)$ & 5 & $(3-7,5)$ & 0,92 \\
\hline \multicolumn{8}{|l|}{ Antecedentes patológicos } \\
\hline Hipertensión & 73 & $(64,04)$ & 19 & $(63,33)$ & 54 & $(64,29)$ & 0,93 \\
\hline Tabaquismo & 40 & $(35,71)$ & 9 & $(31,03)$ & 31 & $(37,35)$ & 0,54 \\
\hline EPOC & 38 & $(33,33)$ & 8 & $(26,67)$ & 30 & $(35,71)$ & 0,37 \\
\hline Diabetes mellitus & 28 & $(24,56)$ & 3 & $(10,00)$ & 25 & $(29,76)$ & 0,03 \\
\hline ECV & 22 & $(19,30)$ & 8 & $(26,67)$ & 14 & $(16,67)$ & 0,23 \\
\hline Enfermedad renal crónica & 19 & $(16,67)$ & 6 & $(20,00)$ & 13 & $(15,48)$ & 0,57 \\
\hline Demencia & 18 & $(15,79)$ & 8 & $(26,67)$ & 10 & $(11,90)$ & $0,08 \mathrm{t}$ \\
\hline Hipotiroidismo & 15 & $(13,16)$ & 2 & $(6,67)$ & 13 & $(15,48)$ & $0,35 \mathrm{t}$ \\
\hline Insuficiencia cardíaca & 13 & $(11,40)$ & 2 & $(6,67)$ & 11 & $(13,10)$ & $0,51 \mathrm{t}$ \\
\hline Dislipidemia & 10 & $(8,77)$ & 3 & $(10,00)$ & 7 & $(8,33)$ & $0,72 \mathrm{t}$ \\
\hline Trastorno de deglución & 7 & $(6,14)$ & 3 & $(10,00)$ & 4 & $(4,76)$ & $0,38 t$ \\
\hline Cáncer & 7 & $(6,14)$ & 3 & $(10,00)$ & 4 & $(4,76)$ & $0,38 \mathrm{t}$ \\
\hline Neumonía previa & 6 & $(5,26)$ & 1 & $(3,33)$ & 5 & $(5,95)$ & $1,00 t$ \\
\hline Epilepsia & 6 & $(5,26)$ & 4 & $(13,33)$ & 2 & $(2,38)$ & $0,04 \mathrm{t}$ \\
\hline Fibrilación auricular & 5 & $(4,39)$ & 2 & $(6,67)$ & 3 & $(3,57)$ & $0,61 \mathrm{t}$ \\
\hline \multicolumn{8}{|l|}{ Variables clínicas } \\
\hline Hipotensión sistólica & 15 & $(13,16)$ & 5 & $(16,67)$ & 0 & $(11,90)$ & $0,54 \mathrm{t}$ \\
\hline Hipoxemia & 54 & $(47,37)$ & 22 & $(73,33)$ & 32 & $(38,10)$ & $<0,01$ \\
\hline Frecuencia cardíaca** & 84 & $(77-98)$ & 81 & $(80-90)$ & 86,5 & $(75-99,5)$ & 0,51 \\
\hline Frecuencia respiratoria** & 19 & $(16-24)$ & 18 & $(16-26)$ & 20 & $(17-23)$ & 0,73 \\
\hline CURB-65** & 2 & $(1-3)$ & 3 & $(2-3)$ & 2 & $(1-3)$ & $<0,01$ \\
\hline \multicolumn{8}{|l|}{ CURB-65 n (\%) } \\
\hline Neumonía leve* & 70 & $(61,40)$ & 11 & $(36,67)$ & 59 & $(70,24)$ & $<0,01$ \\
\hline Neumonía grave & 44 & $(38,60)$ & 19 & $(63,33)$ & 25 & $(29,76)$ & \\
\hline \multicolumn{8}{|l|}{ Tratamiento n (\%) } \\
\hline Recibió antibiótico & 111 & $(97,37)$ & 30( & $(100,00)$ & 81 & $(96,43)$ & $0,57 \mathrm{t}$ \\
\hline Duración (días) ${ }^{\star \star}$ & 10 & $(8-11)$ & & $(7-11)$ & 9 & $(8-10)$ & 0,70 \\
\hline
\end{tabular}

* Media y desviación estándar

** Mediana y rangos intercuartílicos; test exacto de Fisher

Sin riesgo social $<8$ puntos

- Neumonía leve $<3$ puntos

EPOC: enfermedad pulmonar obstructiva crónica; ECV: enfermedad cerebrovascular

En el análisis bivariado las siguientes variables tuvieron una asociación significativa con la letalidad: edad $\geq 90$ años $\left(R R=2,05 ; I_{95 \%}: 1,13-3,70\right.$, índice de Barthel $\left(R R=5,05 ; I_{95 \%}: 1,63-15,64\right)$, escala de Lawton y Brody $\left(R R=4,86 ; I_{95 \%}: 1,57-15,05\right)$, epilepsia ( $\left.R R=2,77 ; I C_{95 \%}: 1,09-36,44\right)$, hipoxemia ( $\left.R R=3,05 ; \mathrm{IC}_{95 \%}: 1,49-5,34\right)$, compromiso multilobar $(\mathrm{RR}=2,77$; $\left.\mathrm{IC}_{95 \%}: 1,43-5,34\right)$, nitrógeno ureico ( $\left.R \mathrm{R}=1,02 ; \mathrm{IC}_{95 \%}: 1,01-1,03\right), \mathrm{BUN} \geq 22,5 \mathrm{mg} /$ dl (RR=5,00; IC $\left.{ }_{95 \%}: 2,06-12,14\right)$, y CURB65 (RR=2,75; IC $\left.{ }_{95 \%}: 1,45-5,21\right)$. Los resultados completos del análisis bivariado se resumen en el cuadro 3 . En el análisis de regresión multivariable, la edad de 90 años o más $(p=0,04)$, un puntaje cero en la escala de Lawton y Brody categorizado como dependencia total $(p=0,04)$, la presencia de compromiso multilobar $(p=0,02)$ y el valor del nitrógeno ureico elevado $(p<0,01)$ se asociaron de manera independiente con la letalidad a los 30 días (cuadro 4). En la figura 2 se observa un aumento de 
la letalidad $(p<0,001)$ y del riesgo relativo con un mayor número de factores de riesgo (edad $\geq 90$ años, compromiso multilobar, puntaje de cero en la escala de Lawton, BUN alto), especialmente con la presencia de, por lo menos, dos factores a la vez.

Cuadro 2. Características funcionales y mentales, y resultados en exámenes paraclínicos de la población de estudio

\begin{tabular}{|c|c|c|c|c|c|c|c|}
\hline \multirow[b]{2}{*}{ Características } & \multicolumn{2}{|r|}{ General } & \multicolumn{4}{|c|}{ Letalidad a $\mathbf{3 0}$ días } & \multirow[b]{2}{*}{$\mathbf{p}$} \\
\hline & \multicolumn{2}{|r|}{ n (\%) } & \multicolumn{2}{|r|}{$\begin{array}{c}\text { Sí } \\
(n=30) \\
n(\%)\end{array}$} & \multicolumn{2}{|r|}{$\begin{array}{c}\text { No } \\
(n=84) \\
n(\%)\end{array}$} & \\
\hline \multicolumn{8}{|l|}{ Índice de Barthel al ingreso } \\
\hline Independiente & 33 & $(28,95)$ & 2 & $(6,67)$ & 31 & $(36,90)$ & $<0,01$ \\
\hline Dependiente & 81 & $(71,05)$ & 28 & $(93,33)$ & 53 & $(63,10)$ & $<0,01$ \\
\hline \multicolumn{8}{|l|}{ Escala de Lawton al ingreso } \\
\hline Sin dependencia total & 40 & $(35,09)$ & 3 & $(10,0)$ & 37 & $(44,0)$ & $<0,01$ \\
\hline Con dependencia total ${ }^{\perp}$ & 74 & $(64,91)$ & 27 & $(90,0)$ & 47 & $(56,0)$ & $<0,01$ \\
\hline \multicolumn{8}{|l|}{ MiniMental } \\
\hline Deterioro cognitivo leve ${ }^{\theta}$ & 63 & $(55,26)$ & 13 & $(43,33)$ & 50 & $(59,52)$ & 0,13 \\
\hline Deterioro cognitivo grave & 51 & $(44,74)$ & 17 & $(56,67)$ & 34 & $(40,48)$ & 0,13 \\
\hline Delirio (CAM) & 45 & $(39,47)$ & 14 & $(46,67)$ & 31 & $(36,90)$ & 0,35 \\
\hline \multicolumn{8}{|l|}{ Variables paraclínicas } \\
\hline Compromiso multilobar & 6 & $(5,26)$ & 4 & $(13,33)$ & 2 & $(2,38)$ & 0,04 \\
\hline Derrame pleural & 29 & $(25,44)$ & 8 & $(26,67)$ & 21 & $(25,00)$ & 0,86 \\
\hline Leucocitos $^{\star *}$ & $11.440(8$ & $8.540-15.230)$ & 10.510 & $3.730-13.250)$ & 11.690 & $(8.525-15.355)$ & 0,49 \\
\hline Hemoglobina** & 11,6 & $65(9,6-12,7)$ & 10,6 & $(9,5-12,4)$ & 11,85 & $(9,95-13,1)$ & 0,26 \\
\hline Albumina** & 2,97 & $7 \quad(2,63-3,3)$ & 2,72 & $(2,34-3,09)$ & 3,01 & $(2,75-3,37)$ & 0,01 \\
\hline Creatinina** & 1,01 & $1 \quad(0,74-1,41)$ & 1,04 & $4 \quad(0,75-1,26)$ & 1,01 & $(0,73-1,48)$ & 0,94 \\
\hline Nitrógeno ureico** & 22,5 & $(17-34)$ & 34 & $(25-54)$ & 20 & $(15-26,5)$ & $<0,01$ \\
\hline Sodio** & 140 & $(137-144,4)$ & 142,63 & $3(137-149,1)$ & 139,83 & $(137-142)$ & 0,1 \\
\hline Proteína $\mathrm{C}$ reactiva** & 73,66 & $6(23,59-140)$ & 85,06 & $6(61,23-129,7)$ & 65,99 & $(19,53-148,83)$ & 0,20 \\
\hline
\end{tabular}

${ }^{*}$ Media y desviación estándar

** Mediana y rangos intercuartílicos; test exacto de Fisher

- Dependiente: $\leq 60$ puntos

${ }^{\perp}$ Con dependencia total: 0 puntos

${ }^{\theta}$ Deterioro cognitivo leve: $>23$ puntos;

CAM: Confusion Assessment Method

Cuadro 3. Regresiones en el análisis log-binomial bivariado de los factores asociados con la letalidad a 30 días

\begin{tabular}{|c|c|c|c|}
\hline Características & $\mathbf{R R}$ & $I C_{95 \%}$ & $\mathbf{p}$ \\
\hline Edad* $^{*}$ & 1,05 & $1,02-1,09$ & $<0,01$ \\
\hline $\mathrm{dad} \geq 90$ &, 05 & & \\
\hline sala de Gijón & & & \\
\hline rthel al ingres & & & \\
\hline 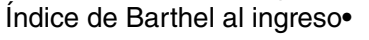 & 5,05 & & 01 \\
\hline 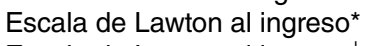 & 0,56 & $0,25-0,89$ & 0,01 \\
\hline Lawton al ingreso ${ }^{\perp}$ & 4,86 & 1,5 & $<0,01$ \\
\hline Minim & 1,61 & 0,8 & 0,13 \\
\hline Diab & 0,34 & 0,1 & 0,0 \\
\hline em & 1,94 & $-3,65$ & 0,04 \\
\hline & 2,77 & $-5,34$ & $<0,01$ \\
\hline & 3,05 & $1,49-6,28$ & $<0,01$ \\
\hline Comr & 2,77 & $1,43-5,34$ & $<0,01$ \\
\hline Albu & 0,46 & $0,27-0,80$ & $<0,01$ \\
\hline Nitróg & 1,02 & $1,01-1,03$ & $<0,01$ \\
\hline Nitrógeno ureico $\geq 22,5 \mathrm{mg} / \mathrm{dl}$ & 5,00 & $2,06-12,14$ & $<0,01$ \\
\hline CUP & 1,46 & $1,16-1,84$ & $<0,01$ \\
\hline CURB-65 $\varnothing$ & 2,75 & $1,45-5,21$ & $<0,01$ \\
\hline
\end{tabular}

* Variables numéricas

- Dependiente: $\leq 60$ puntos

${ }^{\perp}$ Con dependencia total: 0 puntos

${ }^{\varnothing}$ CURB-65 $\geq 3$ puntos

RR: riesgo relativo; IC: intervalo de confianza 
Cuadro 4. Regresión log-binomial múltiple de los factores asociados con la letalidad a 30 días

\begin{tabular}{lccr}
\hline Características & RR & IC $_{95 \%}$ & \multicolumn{1}{c}{$\mathbf{p}$} \\
\hline Escala de Lawton al ingreso: 0 puntos & 3,20 & $1,05-9,78$ & 0,04 \\
Edad $\geq 90$ & 1,62 & $1,05-2,68$ & 0,04 \\
Multilobar & 1,92 & $1,12-3,32$ & 0,02 \\
Nitrógeno ureico $\geq 22,5 \mathrm{mg} / \mathrm{dl}$ & 3,93 & $1,67-9,25$ & $<0,01$ \\
\hline
\end{tabular}

RR: riesgo relativo; IC: intervalo de confianza

La bondad del ajuste se evaluó con la prueba de desviación $(p=0,99)$.

Porcentaje (\%) de letalidad de acuerdo al número de factores de riesgo

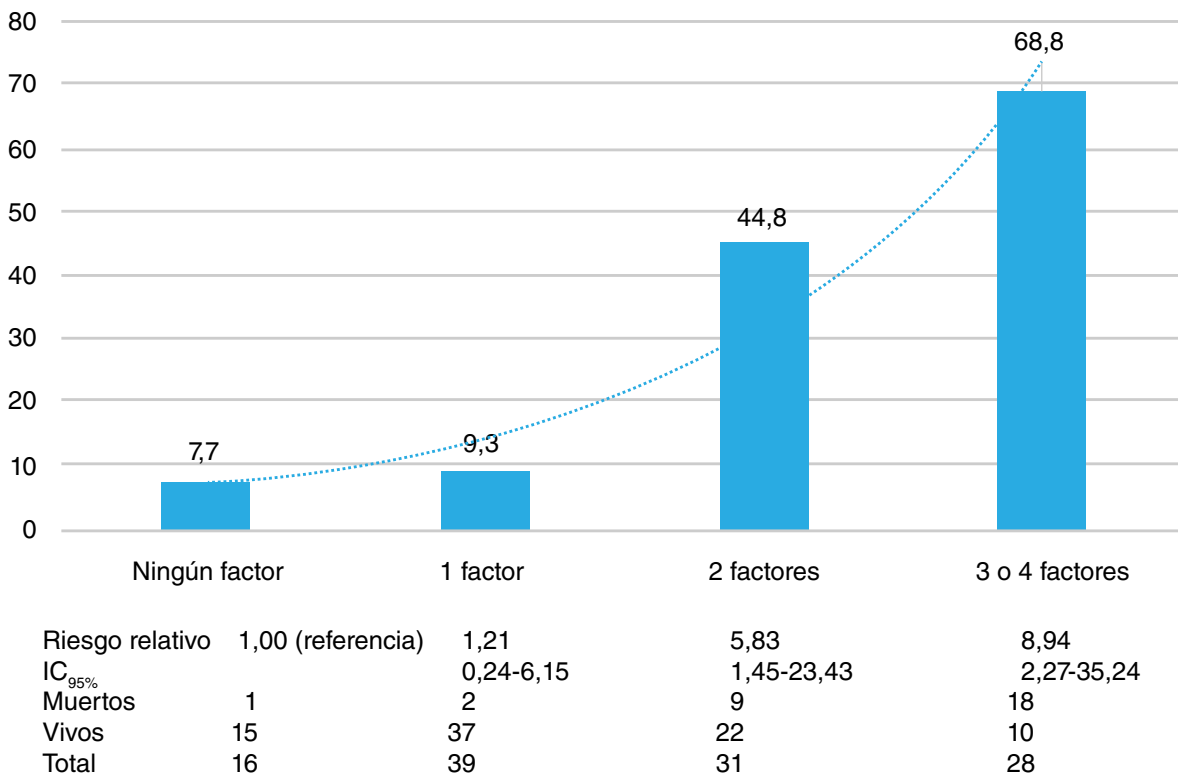

Los factores de riesgo incluyen la edad igual o mayor de 90 años, el compromiso multilobar, puntaje de cero en la escala de Lawton, y BUN alto. Hubo un aumento significativo en las muertes $(p<0,001)$ a medida que el número de factores de riesgo aumentaba $(n=114)$.

Figura 2. Porcentaje de letalidad debido a neumonía según el número de factores de riesgo

\section{Discusión}

En este estudio de pacientes adultos mayores con neumonía hospitalizados en una unidad de atención del paciente geriátrico agudo se encontró que la presencia de BUN elevado ( $\geq 22,5 \mathrm{mg} / \mathrm{dll}$ ), la dependencia total en las actividades instrumentales según la escala de Lawton y Brody al ingreso, el compromiso multilobar y la edad avanzada ( $\geq 90$ años) fueron los principales factores predictivos de letalidad a los 30 días.

El BUN es un parámetro de laboratorio importante en la evaluación del paciente con neumonía; su elevación esta mediada por la hipoperfusión renal que genera la activación del sistema renina-angiotensina-aldosterona, la hormona arginina-vasopresina y el sistema simpático, generando una reabsorción de sodio, agua y urea (33). Es una de las variables de las escalas CURB65 y PSI, y aunque se ha descrito su peso en la asociación con la letalidad, su relevancia en el paciente adulto mayor es controversial. A pesar de que la descripción de los niveles de BUN es frecuente en los estudios, la mayoría no ha demostrado una asociación estadísticamente independiente con la letalidad a corto plazo $(19-21,23,24)$. 
Sin embargo, los resultados de dos cohortes, una retrospectiva procedente de Singapur y otra prospectiva de España, publicados en el 2018 respaldaban la asociación con esta variable. En el primer estudio se evaluaron 1.902 pacientes según su grupo etario y se encontró que en participantes entre los 65 y 84 años los niveles de BUN superiores a $31 \mathrm{mg} / \mathrm{dl}$ tenían una asociación con la mortalidad a 30 días $\left(\mathrm{OR}=2,0 ; \mathrm{IC}_{95 \%}: 1,3-3,1\right)$, hecho que se replicó, además, en la población mayor de 84 años (34). En el segundo estudio, de Uranga, et al., en participantes con una media de edad de 69 años, de los cuales el 33 $\%$ era mayor de 80 años, se demostró que un BUN de más de $30 \mathrm{mg} / \mathrm{dl}$ era una variable con asociación estadística con la mortalidad a un año a partir del episodio de neumonía (HR=1,55; IC $\left.\mathrm{C}_{95 \%}: 1,07-2,24\right)(35)$. En la población del presente estudio se validaron estos resultados al demostrar que un BUN de $22,5 \mathrm{mg} / \mathrm{dl}$ o más en el momento de ingreso se relacionaba con un aumento mayor a tres veces del riesgo de muerte a los 30 días en pacientes adultos mayores con neumonía ( $\left.\mathrm{RR}=3,93 ; \mathrm{IC}_{95 \%}: 1,67-9,25\right)$.

La discapacidad como la imposibilidad total o parcial de participar en las actividades de la vida diaria se atribuye al efecto negativo de la interacción entre el paciente anciano y el medio ambiente. La discapacidad está asociada con el incremento en el costo de la asistencia sanitaria (36), la disminución de la calidad de vida (37) y la mortalidad. En el 2016 un estudio retrospectivo en 1.834 pacientes entre los 60 y los 84 años demostró que la discapacidad aumentaba dos veces el riesgo de muerte por cualquier causa (38). En el presente estudio la segunda variable con mayor magnitud de asociación con el resultado primario fue la pérdida de la funcionalidad en las actividades instrumentales medida por la escala de Lawton y Brody al ingreso, donde un puntaje de 0 indicaba un riesgo tres veces mayor de letalidad a los 30 días después de un episodio de neumonía.

Este hecho se documentó previamente en el 2014 en un estudio de Calle, et al., en 456 pacientes de 75 años de edad o más, en quienes se demostró que conservar el dominio funcional ( $>5$ puntos en la escala de Lawton y Brody) se relacionaba de manera independiente con una menor letalidad a corto plazo, configurándose como un factor protector $\left(\mathrm{OR}=0,09 ; \mathrm{IC}_{95 \%}: 0,01-\right.$ $0,81)(21)$. Ello se explicaría por la tendencia acelerada de envejecimiento en la población y el incremento en la expectativa de vida asociado con la pérdida paulatina de la funcionalidad, que empieza a cobrar relevancia con las limitaciones para llevar a cabo las actividades instrumentales. En el análisis bivariado en la cohorte del presente estudio, un puntaje menor de 60 (paciente dependiente) en el IBi fue un factor de riesgo de letalidad a los 30 días ( $\left.R R=5,05 ; I_{95 \%}: 1,63-15,64\right)$, sin embargo, su significación estadística no se mantuvo en el análisis multivariable. Este hecho contrasta con los resultados obtenidos en estudios previos, en los que una dependencia funcional moderada o grave en las actividades básicas se asoció con un riesgo tres veces mayor de letalidad a corto plazo (22), y un índice de Barthel menor de 40 puntos se asoció con la letalidad hospitalaria a tres meses y a un año, con un OR de 9,45, 6,84 y 19,55, respectivamente, determinando el impacto de la discapacidad a corto y largo plazo (39).

El compromiso de dos o más lóbulos en la radiografía de tórax se ha descrito tradicionalmente como un factor que contribuye a la hospitalización y el riesgo de muerte por neumonía, aunque con un peso secundario en la magnitud del riesgo comparado con los parámetros fisiológicos. Un metaanálisis del 2013 en el que se evaluó el impacto de la neumonía multilobar en 11.456 pacientes de 22 estudios, la mayoría prospectivos, 
demostró que este hallazgo radiográfico es un factor de riesgo de letalidad, con un RR de 2,57 (40). El presente estudio corrobora este hallazgo en la población adulta mayor, siendo el compromiso multilobar la tercera variable estadísticamente relacionada con la letalidad a corto plazo, con un RR de 1,92. Resultados similares se obtuvieron en una cohorte del 2014 (21).

En el siglo XIX, antes de la era antimicrobiana, la neumonía fue descrita por Sir William Osler como una enfermedad fatal en el paciente anciano. En la actualidad, a pesar del tratamiento efectivo y el diagnóstico temprano, la tasa de letalidad en la neumonía sigue siendo alta. Según la edad y la gravedad de la enfermedad, puede variar entre el 4,9 y el $48 \%$ (41). En la presente cohorte la tasa de letalidad a 30 días fue de $26,3 \%$, lo que la sitúa en el límite superior del rango descrito en la literatura (6,8 a 28,7\%). Este alto porcentaje puede explicarse por la edad cronológica avanzada, de 84,5 años en promedio, superior a la de otros estudios $(22,39)$. En estudios previos se ha demostrado el impacto de la edad en la letalidad, incluso en cohortes de pacientes muy ancianos, y se ha estimado el aumento del riesgo de muerte en adultos con edades de 80 años o más $\left(O R=2,10 ; I_{95 \%}: 1,40-3,15\right)(35)$, de 85 o más $\left(\mathrm{OR}=3,01 ; \mathrm{IC}_{95 \%}: 1,71-5,30\right)(22)$ y de 90 años o más $(\mathrm{OR}=3,10$; $\left.\mathrm{IC}_{95 \%}: 1,31-7,36\right)(21)$. Este hecho se corroboró en nuestra población al demostrar que una edad de 90 años o más confiere un RR de 1,62 asociado con la letalidad a 30 días.

Como se ha descrito en la literatura especializada, la enfermedad cerebrovascular, la enfermedad pulmonar, el tabaquismo, la enfermedad renal crónica y la hipertensión son comorbilidades frecuentes (2), aunque sin significación estadística en términos de asociación con la letalidad a 30 días. Varios autores han encontrado que la alteración del estado de conciencia constituye un predictor de la letalidad $(20,22)$ y, aunque en el presente estudio no hubo diferencias en esta variable y los pacientes que presentaron el resultado primario tenían un $10 \%$ más de alteración del estado de conciencia, posiblemente el tamaño de la muestra y el delirio como criterio de ingreso en la unidad geriátrica de agudos atenuaron el resultado. La hipoxemia en el adulto mayor es una de las variables clínicas relevantes en el diagnóstico de neumonía y, aunque en un estudio fue descrita como un factor de riesgo de letalidad, con un OR de 2,7 (34), en nuestro estudio no se demostró la asociación independiente con la muerte.

Se ha demostrado que los sistemas de puntuación establecidos para determinar la gravedad de la neumonía y el sitio de manejo ideal, el CURB65 y Pneumonia Severity Index (PSI) no tienen la suficiente precisión para clasificar al paciente adulto mayor (2). El alto valor que da el PSI a la edad y las comorbilidades suele sobreestimar el riesgo, en tanto que la ausencia de la carga de enfermedad como variable en la escala CURB65 puede subestimar el riesgo en los pacientes adultos mayores, que pueden descompensarse de manera significativa, incluso cuando se trata de neumonías catalogadas como leves en dicha escala. Un estudio del 2010 que incluyó tres grupos etarios (18-64, 65-84, y $\geq 85$ años) corroboró este hallazgo al demostrar un poder discriminativo decreciente del PSI y la CURB-65 con respecto a las categorías de edad avanzada, con un área bajo la curva en la CURB65 de 0,80, 0,73 y 0,6 en cada grupo, respectivamente (42). En nuestra población, un puntaje en la escala CURB65 mayor o igual a 3 implicaba un mayor riesgo de letalidad a corto plazo, con un RR de 2,75, aunque esta asociación no fue relevante en el análisis multivariado. 
Por último, los resultados demuestran la importancia de abordar la neumonía en el adulto mayor (incluso desde el ingreso en el servicio de urgencias) con un enfoque multidimensional; en nuestra cohorte una variable sociodemográfica, una de laboratorio, una de imagenología y una funcional explicaron la letalidad a corto plazo. El modelo presentado evidenció que las variables descritas tuvieron un efecto aditivo en el riesgo de letalidad a 30 días, por lo que tener dos o tres o más variables incrementó más de 5 y 8 veces la posibilidad de un desenlace adverso, respectivamente. El objetivo con estos resultados es cambiar la perspectiva en la atención del adulto mayor. Una adecuada clasificación del paciente en cuanto al riesgo permite tomar decisiones acertadas sobre el manejo integral, lo que se traduce en una reducción de la letalidad de una enfermedad frecuente que cobra miles de víctimas cada año alrededor del mundo.

La fortaleza del presente estudio reside en que los casos de neumonía habían sido confirmados por radiología en una población con criterios de selección estrictos que permitieron determinar los factores con impacto en la letalidad a 30 días en pacientes hospitalizados en una unidad de atención del paciente geriátrico agudo, población con altas tasas de morbimortalidad que poco se ha considerado en los estudios. Además, la cohorte analizada estaba internada en un servicio especializado de hospitalización geriátrica con funciones académicas y asistenciales a cargo de un equipo interdisciplinario. Además, para garantizar la calidad de la información cada escala aplicada y cada dato obtenido fue responsabilidad de profesionales entrenados durante años en el área específica (médicos y profesionales de rehabilitación, psicología y trabajo social).

Una de las limitaciones fue la información secundaria y el posible sesgo de selección, ya que se excluyeron pacientes con mayor dependencia funcional y aquellos cuyos datos estaban incompletos, pues esto afecta la generalización de los resultados. Asimismo, el tamaño de la muestra pudo atenuar la magnitud de la asociación de algunas variables; además, la condición retrospectiva puede incrementar el error de medición, pues no hay control sobre las fuentes de información y es difícil ajustar por otros factores predictores durante el análisis. Tampoco se pudo incluir el dato de la procedencia (hogar familiar Vs. hogar geriátrico) debido a la información incompleta.

Es importante destacar que se trata de una cohorte de adultos mayores con una media de edad avanzada, de 84 años, y con al menos una comorbilidad, lo cual limita la validez externa de los resultados en otros pacientes que cumplan con las características planteadas de la población objetivo del presente estudio. Estas limitaciones motivan la realización de estudios de cohorte prospectiva y preferiblemente multicéntricos que permitan establecer otros factores potencialmente modificables que afectan la supervivencia de los pacientes.

En los pacientes ancianos hospitalizados por neumonía en esta unidad de atención del paciente geriátrico agudo, la edad muy avanzada ( $\geq 90$ años), la presencia de compromiso multilobar, la dependencia total en el estado funcional (según la escala de Lawton y Brody al ingreso) y los niveles de nitrógeno ureico elevados fueron los principales predictores de riesgo de letalidad a corto plazo. Estas variables deben ser evaluadas en el momento de ingreso de pacientes adultos mayores con neumonía para caracterizar mejor el riesgo y definir intervenciones que favorezcan su funcionalidad y supervivencia. 


\section{Referencias}

1. Montufar FE, Varón F, Giraldo L, Sáenz O, Rodríguez A, Alarcón A. Recomendaciones para el diagnóstico, tratamiento y prevención de la neumonía adquirida en la comunidad en adultos. Infectio. 2013;17(Supl 1):1-38. https://doi.org/10.1111/imj.12258

2. Henig O, Kaye KS. Bacterial pneumonia in older adults. Infect Dis Clin North Am. 2017;31:689-713. https://doi.org/10.1016/j.arbres.2015.12.006

3. Prina E, Ranzani OT, Torres A. Community-acquired pneumonia. Lancet. 2015;386:1097-108. https://doi.org/10.1016/S0140-6736(15)60733-4

4. Pan American Health Organization (PAHO). Estado de salud de la población. Salud del adulto mayor. 2017. Fecha de consulta: 17 de julio de 2020. Disponible en: https://www.paho. org/salud-en-las-americas-2017/?post t es=salud-del-adulto-mayor\&lang=es

5. Commission Européenne. The 2015 Ageing Report: Economic and budgetary projections for the 28 EU Member States (2013-2060). Fecha de consulta: 17 de julio de 2020. Disponible en: http://ec.europa.eu/economy finance/publications/european economy/2015/ee3 en.htm

6. Ministerio de Salud, Colombia. Envejecimiento demográfico. Colombia 1951-2020. Fecha de consulta: 23 de julio de 2020. Disponible en: https://www.minsalud.gov.co/sites/rid/Lists/ BibliotecaDigital/RIDE/DE/PS/Envejecimiento-demografico-Colombia-1951-2020.pdf

7. Tichopad A, Roberts C, Gembula I, Hajek P, Skoczynska A, Hryniewicz W, et al. Clinical and economic burden of community-acquired pneumonia among adults in the Czech Republic, Hungary, Poland and Slovakia. PLoS One. 2013;8:1-7. https://doi.org/10.1371/journal.pone.0071375

8. Yu H, Rubin J, Dunning S, Li S, Sato R. Clinical and economic burden of communityacquired pneumonia in the medicare fee-for-service population. J Am Geriatr Soc. 2012;60:2137-43. https://doi.org/10.1111/j.1532-5415.2012.04208.x

9. Brown JD, Harnett J, Chambers R, Sato R. The relative burden of community-acquired pneumonia hospitalizations in older adults: A retrospective observational study in the United States. BMC Geriatr. 2018;18:1-11. https://doi.org/10.1186/s12877-018-0787-2

10. Buzzo AR, Roberts C, Mollinedo LG, Quevedo JM, Casas GL, Soldevilla JM. Morbidity and mortality of pneumonia in adults in six Latin American countries. Int $\mathrm{J}$ Infect Dis. 2013;17:e673-7. https://doi.org/10.1016/j.jijid.2013.02.006

11. Mattila JT, Fine MJ, Limper AH, Murray PR, Chen BB, Lin PL. Pneumonia: Treatment and diagnosis. Ann Am Thorac Soc. 2014;11:S189-92. https://doi.org/10.1513/AnnalsATS.201401-027PL

12. Fry AM, Shay DK, Holman RC, Curns AT, Anderson LJ. Trends in hospitalizations for pneumonia among persons aged 65 years or older in the United States, 1988-2002. J Am Med Assoc. 2005;294:2712-9. https://doi.org/10.1001/jama.294.21.2712

13. Departamento Administrativo Nacional de Estadística (DANE). Estadísticas vitales año 2016 - 2017. Fecha de consulta: 9 de diciembre de 2019. Disponible en: https://www.dane.gov.co/ files/investigaciones/poblacion/bt estadisticasvitales_2016def-2017pre.pdf

14. Montúfar FE, Rueda ZV, Correa LT, Ortega H, Ortega J, Segura A, et al. Características y comportamiento de la neumonía adquirida en la comunidad (NAC) en adultos mayores ( $\geq$ 65 años) hospitalizados en el Valle de Aburrá, Antioquia, Colombia. Infectio. 2006;10:113.

15. Romero-Rizos L, Sánchez-Jurado PM, Abizanda-Soler P. El anciano en la unidad de geriatría de agudos. Rev Esp Geriatr Gerontol. 2009;44(Supl. 1):15-26. https://doi.org/10.1016/j.regg.2009.02.006

16. Fox MT, Persaud M, Maimets I, O'Brien K, Brooks D, Tregunno D, et al. Effectiveness of acute geriatric unit care using acute care for elders components: A systematic review and meta-analysis. J Am Geriatr Soc. 2012;60:2237-45. https://doi.org/10.1111/jgs.12028

17. Goh KS, Low SK, Zhang D, Png GK, Lin H, Ang WS, et al. Mortality predictors in an acute care geriatric unit in Singapore. Proc Singapore Healthc. 2018;27:265-9. https://doi.org/10.1177/2010105818762915

18. Neupane B, Walter SD, Krueger P, Marrie T, Loeb M. Predictors of inhospital mortality and re-hospitalization in older adults with community-acquired pneumonia: A prospective cohort study. BMC Geriatr. 2010;10. https://doi.org/10.1186/1471-2318-10-22

19. Ma HM, Tang WH, Woo J. Predictors of in-hospital mortality of older patients admitted for community-acquired pneumonia. Age Ageing. 2011;40:736-41. https://doi.org/10.1093/ageing/afr087 
20. Guzzi L, Lopardo G, Espinola L, Barcelona L. Mortalidad en pacientes con neumonía provenientes de geriátrico: estudio caso-control. Rev Am Med Respir. 2013;13:133-9.

21. Calle A, Márquez MA, Arellano M, Pérez LM, Pi-Figueras M, Miralles R. Valoración geriátrica y factores pronósticos de mortalidad en pacientes muy ancianos con neumonía extrahospitalaria. Arch Bronconeumol. 2014;50:429-34. https://doi.org/10.1016/j.arbres.2014.01.012

22. Torner N, Izquierdo C, Soldevila N, Toledo D, Chamorro J, Espejo E, et al. Factors associated with 30-day mortality in elderly inpatients with community acquired pneumonia during 2 influenza seasons. Hum Vaccines Immunother. 2017;13:450-5. https://doi.org/10.1080/21645515.2017.1264782

23. Falcone M, Russo A, Gentiloni Silverj F, Marzorati D, Bagarolo R, Monti M, et al. Predictors of mortality in nursing-home residents with pneumonia: A multicentre study. Clin Microbiol Infect. 2018;24:72-7. https://doi.org/10.1016/j.cmi.2017.05.023

24. Pieralli F, Vannucchi V, De Marzi G, Mancini A, Bacci F, Para O, et al. Performance status and in-hospital mortality of elderly patients with community acquired pneumonia. Intern Emerg Med. 2018;13:501-7. https://doi.org/10.1007/s11739-018-1822-1

25. Charlson ME, Pompei P, Ales KL MC. A new method of classifying prognostic comorbidity in longitudinal studies: Development and validation. J Chronic Dis. 1987;40:373-83. https://doi.org/10.1016/0021-9681(87)90171-8

26. Lim WS, Van Der Eerden MM, Laing R, Boersma WG, Karalus N, Town Gl, et al. Defining community acquired pneumonia severity on presentation to hospital: An international derivation and validation study. Thorax. 2003;58:377-82. https://doi.org/ 10.1136/thorax.58.5.377

27. Granger CV, Albretch GL, Hamilton BB. Outcome of comprehensive medical rehabilitation: Measurement by PULSES profile and the Barthel index. Arch Phys Med Rehabil. 1979;60:145-54.

28. Lawton MP, Brody EM. Assessment of older people: Self-maintaining and instrumental activities of daily living. Gerontologist. 1969;9:179-86.

29. Inouye SK, Van Dyck CH, Alessi CA, Balkin S, Siegal AP, Horwitz RI. Clarifying confusion: The confusion assessment method: A new method for detection of delirium. Ann Intern Med. 1990;113:941-8. https://doi.org/10.7326/0003-4819-113-12-941

30. Folstein MF, Folstein SE, McHugh PR. "Mini-mental state." A practical method for grading the cognitive state of patients for the clinician. J Psychiat Res. 1975;12:189-98. https://doi.org/10.1016/0022-3956(75)90026-6

31. Greenland S. Modeling and variable selection in epidemiologic analysis. Am J Public Health. 1989;79:340-9. https://doi.org/10.2105/ajph.79.3.340

32. Triana-Cuervo PA, Ocampo-Chaparro JM, Reyes-Ortiz CA, Casanova ME. Tasa de filtración glomerular estimada y mortalidad posterior al alta en una unidad geriátrica de agudos. Rev Esp Geriatr Gerontol. 2019;54:265-71. https://doi.org/10.1016/j.regg.2019.04.006

33. Beier K, Eppanapally S, Bazick HS, Chang D, Mahadevappa K, Gibbons FK, et al. Elevation of blood urea nitrogen is predictive of long-term mortality in critically ill patients independent of normal creatinine. Crit Care Med. 2011;39:305-13. https://doi.org/10.1097/CCM.0b013e3181ffe22a

34. Zhang ZX, Yong Y, Tan WC, Shen L, Ng HS, Fong KY. Prognostic factors for mortality due to pneumonia among adults from different age groups in Singapore and mortality predictions based on PSI and CURB-65. Singapore Med J. 2018;59:190-8. https://doi.org/10.11622/smedi.2017079

35. Uranga A, Quintana JM, Aguirre U, Artaraz A, Diez R, Pascual S, et al. Predicting 1-year mortality after hospitalization for community-acquired pneumonia. PLoS One. 2018;13:e0192750. https://doi.org/10.1371/journal.pone.0192750

36. Fried LP, Ferrucci L, Darer J, Williamson JD, Anderson G. Untangling the concepts of disability, frailty, and comorbidity: Implications for improved targeting and care. J Gerontol A Biol Sci Med Sci. 2004;59:M255-63. https://doi.org/10.1093/gerona/59.3.m255

37. Motl RW, McAuley E. Physical activity, disability, and quality of life in older adults. Phys Med Rehabil Clin N Am. 2010;21:299-308. https://doi.org/10.1016/j.pmr.2009.12.006

38. Wu LW, Chen WL, Peng TC, Chiang ST, Yang HF, Sun YS, et al. All-cause mortality risk in elderly individuals with disabilities: A retrospective observational study. BMJ Open. 2016;6. https://doi.org/10.1136/bmjopen-2016-011164 
39. Corrao S, Argano C, Natoli G, Nobili A, Corazza GR, Mannucci PM, et al. Disability, and not diabetes, is a strong predictor of mortality in oldest old patients hospitalized with pneumonia. Eur J Intern Med. 2018;54:53-9. https://doi.org/ 10.1016/j.ejim.2018.04.012

40. Mannu GS, Loke YK, Curtain JP, Pelpola KN, Myint PK. Prognosis of multi-lobar pneumonia in community-acquired pneumonia: A systematic review and meta-analysis. Eur J Intern Med. 2013;24:857-63. https://doi.org/10.1016/j.ejim.2013.05.001

41. Zalacaín R, Torres A, Celis R, Blanquer J, Aspa J, Esteban L, et al. Community-acquired pneumonia in the elderly: Spanish multicentre study. Eur Respir J. 2003;21:294-302. https://doi.org/10.1183/09031936.03.00064102

42. Chen JH, Chang SS, Liu JJ, Chan RC, Wu JY, Wang WC, et al. Comparison of clinical characteristics and performance of pneumonia severity score and CURB-65 among younger adults, elderly and very old subjects. Thorax. 2010;65:971-7.

https://doi.org/10.1136/thx.2009.129627 